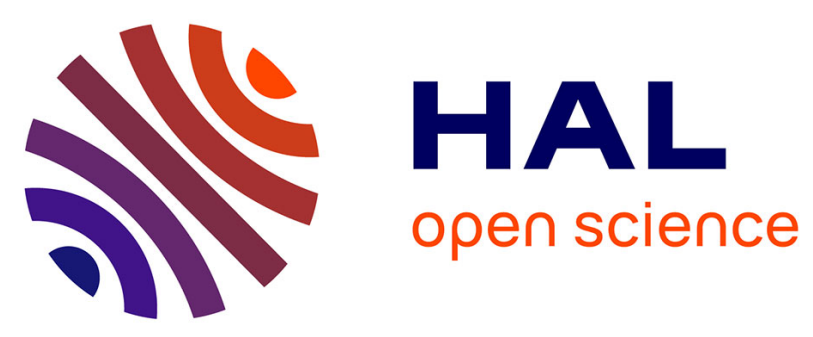

\title{
Multi-Sensor PHD: Construction and Implementation by Space Partitioning
}

\author{
Emmanuel Delande, Emmanuel Duflos, Philippe Vanheeghe, Dominique \\ Heurguier
}

\section{- To cite this version:}

Emmanuel Delande, Emmanuel Duflos, Philippe Vanheeghe, Dominique Heurguier. Multi-Sensor PHD: Construction and Implementation by Space Partitioning. International Conference on Acoustics, Speech and Signal Processing (ICASSP), 2011, May 2011, Prague, Czech Republic. pp.3632 - 3635, 10.1109/ICASSP.2011.5947137 • hal-00639724

\section{HAL Id: hal-00639724}

\section{https://hal.inria.fr/hal-00639724}

Submitted on 9 Nov 2011

HAL is a multi-disciplinary open access archive for the deposit and dissemination of scientific research documents, whether they are published or not. The documents may come from teaching and research institutions in France or abroad, or from public or private research centers.
L'archive ouverte pluridisciplinaire HAL, est destinée au dépôt et à la diffusion de documents scientifiques de niveau recherche, publiés ou non, émanant des établissements d'enseignement et de recherche français ou étrangers, des laboratoires publics ou privés. 


\title{
MULTI-SENSOR PHD: CONSTRUCTION AND IMPLEMENTATION BY SPACE PARTITIONING
}

\author{
$\begin{array}{lll}\text { E. DELANDE } & \text { E. DUFLOS } \\ & \text { P. VANHEEGHE }\end{array} \quad$ D. HEURGUIER ${ }^{\dagger}$ \\ LAGIS FRE CNRS 3303 * \\ Ecole Centrale de Lille \\ 59651 Villeneuve d'Ascq, France \\ SEA ${ }^{\dagger}$ \\ Thales Communication \\ 92704 Colombes, France
}

\begin{abstract}
The Probability Hypothesis Density (PHD) is a well-known method for single-sensor multi-target tracking problems in a Bayesian framework, but the extension to the multi-sensor case seems to remain a challenge. In this paper, an extension of Mahler's work to the multi-sensor case provides an expression of the true PHD multi-sensor data update equation. Then, based on the configuration of the sensors' fields of view (FOVs), a joint partitioning of both the sensors and the state space provides an equivalent yet more practical expression of the data update equation, allowing a more effective implementation in specific FOV configurations.
\end{abstract}

Index Terms-Probability Hypothesis Density, Multi-sensor system, Multi-target tracking

\section{INTRODUCTION}

In the general multi-sensor multi-target Bayesian framework, an unknown (and possibly varying) number of targets whose states $x_{1}, \ldots x_{n}{ }^{1}$ are observed by several sensors which produce a collection of measurements $z_{1}, \ldots, z_{m}$ at every time step $k$. Well-known models to this problem are track-based models such as the joint probability data association (JPDA) [2] or joint multi-target probabilities such as the joint multi-target probability density (JMPD) [3]. This paper is based on Mahler's work on finite sets statistics (FISST) [4] which provides a mathematical framework to build multi-object densities and derive the Bayesian rules. Randomness on object number and their states are encapsulated into random finite sets (RFS), namely multi-target (state) sets $X=\left\{x_{1}, \ldots, x_{n}\right\}$ and multi-sensor (measurement) set $Z_{k}=\left\{z_{1}, \ldots, z_{m}\right\}$. The objective is then to propagate the multi-target probability density $f_{k \mid k}\left(X \mid Z^{(k)}\right)$ by using the Bayesian set equations at every time step $k$ :

$$
\begin{aligned}
f_{k+1 \mid k}\left(X \mid Z^{(k)}\right) & =\int f_{k+1 \mid k}(X \mid W) f_{k \mid k}\left(W \mid Z^{(k)}\right) \delta W \\
f_{k+1 \mid k+1}\left(X \mid Z^{(k+1)}\right) & =\frac{f_{k+1}\left(Z_{k+1} \mid X\right) f_{k+1 \mid k}\left(X \mid Z^{(k)}\right)}{\int f_{k+1}\left(Z_{k+1} \mid W\right) f_{k+1 \mid k}\left(W \mid Z^{(k)}\right) \delta W}
\end{aligned}
$$

where $Z^{(k)}=\bigcup_{t \leqslant k} Z_{t}$ is the collection of measurements up to time $k, f_{k \mid k}\left(W \mid Z^{(k)}\right)$ is the current multi-target posterior density in set $W, f_{k+1 \mid k}(X \mid W)$ is the current multi-target Markov transition

This work was supported by the Direction Générale de l'Armement (DGA) and the Centre National de la Recherche Scientifique (CNRS)

${ }^{1}$ The target state $x_{i} \in \mathcal{X}$ is usually composed of a position, a velocity, density, from set $W$ to set $X, f_{k+1}(Z \mid X)$ is the current multi-sensor multi-target likelihood function.

Although the FISST framework provides set calculus rules, set integrals as well as set probability densities are widely impractical and equations (1), (2) are generally untractable. Mahler then introduced the PHD ([5]), defined on single-target state space $\mathcal{X}$ as follows:

Definition 1.1. The PHD $D_{k \mid k}\left(x \mid Z^{(k)}\right)$ is the quantity whose integral $\int_{S} D_{k \mid k}\left(x \mid Z^{(k)}\right) d x$ on any region $S \subset \mathcal{X}$ is the expected number $N_{k \mid k}(S)=\int|X \cap S| f_{k \mid k}\left(X \mid Z^{(k)}\right) \delta X$ of targets inside $S$.

Mahler proved ([5]) that the PHD $D_{k \mid k}\left(x \mid Z^{(k)}\right)$ is the first-moment density of the multi-target probability density $f_{k \mid k}\left(X \mid Z^{(k)}\right)$. Although defined on single-state space $\mathcal{X}$, the PHD encapsulates information on both target number and states. Assuming that propagating the PHD is sufficient enough for an accurate estimation of target number and target states, the challenge is to find the PHD-equivalent of Bayesian set equations (1) and (2) in order to propagate singletarget moments $D_{k+1 \mid k}\left(x \mid Z^{(k)}\right)$ and $D_{k+1 \mid k+1}\left(x \mid Z^{(k+1)}\right)$ rather than multi-target probability densities.

Under certain assumptions on the target motion model, Mahler provided ([5]) a tractable PHD-equivalent of the time update set equation (1). Likewise, under certain assumptions on the observation model which will be detailed later (section 2.1), the PHD-equivalent of the data update equation (2) is given in [5] for the single-sensor case, in [7], [8] for the two-sensor case. In this paper, the authors first propose an expression of the data update equation in the multi-sensor case and then a partition method in order to lighten the computational cost of the data update step, depending upon the configuration of the sensors' FOVs. The results are more detailed in [1]. A full understanding of this work requires some knowledge about FISST theory and calculus rules which may found in [4].

\section{MULTI-SENSOR DATA UPDATE EQUATION}

Following the time update step and with the same assumption than exposed by Mahler ([5]), the updated distribution $f_{k+1 \mid k}\left(X \mid Z^{(k)}\right)$ is assumed Poisson with parameter $\mu$ and intensity $\mu s(x)^{2}$. Since $f_{k+1 \mid k}\left(X \mid Z^{(k)}\right)$ is Poisson, its intensity $\mu s(x)$ equals the time updated PHD $D_{k+1 \mid k}\left(x \mid Z^{(k)}\right)$ ([5]). Note that the following notations were chosen as close as possible to Mahler's work for clarity's sake.

\footnotetext{
sake

${ }^{2} \mu s()=.\mu_{k+1 \mid k} s_{k+1 \mid k}($.$) , time subscripts are omitted for simplicity's$
} 


\subsection{Observation model}

Assume that, following target transition between time steps $k$ and $k+1$, each sensor $j \in[1 N]$ produces measurements independently of the others according to the observation model described as follows:

- Target $i$ is detected with probability $p_{d}^{[j]}\left(x_{k+1}^{i}\right)^{3}$;

- If detected, target $i$ produces a single measurement $z \in \mathcal{Z}^{[j]}$ with probability distribution $f_{k+1}^{O,[j]}\left(z \mid x_{k+1}^{i}\right)=L_{z}^{[j]}\left(x_{k+1}^{i}\right)$;

- False alarms are Poisson with parameter $\lambda^{[j]}$ and intensity $\lambda^{[j]} c^{[j]}(z)$;

- Observation processes on each target are independent conditionally on the multi-target set $X_{k+1}$.

\subsection{Cross-terms}

Generalizing the single-sensor case led the authors to the introduction and the definition of the cross-terms which played an important role in the construction of the multi-sensor data update equation:

Definition 2.1. For each sensor $j \in[1 N]$, let $g^{[j]}$ be a realvalued function on observation space $\mathcal{Z}^{[j]}$ such that $\forall z \in \mathcal{Z}^{[j]}$, $0 \leqslant g^{[j]}(z) \leqslant 1$. Let $h$ be a real-valued function on state space $\mathcal{X}$ such that $\forall x \in \mathcal{X}, 0 \leqslant h(x) \leqslant 1$. The cross-term $\beta\left[g^{[1]}, \ldots, g^{[N]}, h\right]$ is the functional defined by:

$$
\begin{aligned}
\beta\left[g^{[1]}, \ldots, g^{[N]}, h\right] & =\sum_{j=1}^{N}\left(\lambda^{[j]} c^{[j]}\left[g^{[j]}\right]-\lambda^{[j]}\right) \\
& +\mu s\left[h \prod_{j=1}^{N}\left(1-p_{d}^{[j]}+p_{d}^{[j]} p_{g^{[j]}}^{O,[j]}\right)\right]-\mu
\end{aligned}
$$

where $p_{g^{[j]}}^{O,[j]}(x)=\int g^{[j]}(z) f_{k+1}^{O,[j]}(z \mid x) d z$,

$c^{[j]}\left[g^{[j]}\right]=\int g^{[j]}(z) c^{[j]}(z) d z$ and $s[h]=\int h(x) s(x) d x$.

Using FISST calculus rules ([5]), the cross-term $\beta$ can be differentiated on a single-target space point $x \in \mathcal{X}$ and/or a combination of various single-sensor observation points $z^{[j]} \in \mathcal{Z}^{[j]}$ ([1]). The analytical expressions of the differentiated cross-terms allows an intuitive interpretation as "likelihoods" ${ }^{4}$; for example, with $N=3$ :

- $\left[\frac{\delta \beta\left[g^{[1]}, g^{[2]}, g^{[3]}, h\right]}{\delta x}\right]_{g^{[i]}=0, h=1}=\mu s(x) \prod_{j=1}^{3}\left(1-p_{d}^{[j]}(x)\right): \mathrm{a}$ target is in state $x$ and was not detected by any sensor;

- $\left[\frac{\delta^{3} \beta\left[g^{[1]}, g^{[2]}, g^{[3]}, h\right]}{\delta x \delta z^{[1]} \delta z^{[2]}}\right]_{g^{[i]}=0, h=1}=$ $\mu s(x) \prod_{j=1}^{2}\left(p_{d}^{[j]}(x) L_{z^{[j]}}^{[j]}(x)\right)\left(1-p_{d}^{[3]}(x)\right):$ a target is in state $x$, generated measurements $z^{[1]}$ and $z^{[2]}$ and was not detected by sensor 3 ;

- $\left[\frac{\delta^{2} \beta\left[g^{[1]}, g^{[2]}, g^{[3]}, h\right]}{\delta z^{[1]} \delta z^{[2]}}\right]_{g^{[i]}=0, h=1}=$ $\mu s\left[\prod_{j=1}^{2}\left(p_{d}^{[j]} L_{z^{[j]}}^{[j]}\right)\left(1-p_{d}^{[3]}\right)\right]:$ a single target generated measurements $z^{[1]}$ and $z^{[2]}$ and was not detected by sensor 3 .

Each cross-term above denotes a "likelihood" of a "link" between points in the single-state space $\mathcal{X}$ and/or the observation spaces $\mathcal{Z}^{[j]}$, hence their name.

${ }^{3} p_{d}^{[j]}()=.p_{d, k+1}^{[j]}($.$) , time subscripts are omitted for simplicity's sake$

4"Likelihood" should not be interpreted in its classical Bayesian sense

\subsection{Data update equation}

The authors then constructed the data update equation using the differentiated cross-terms (see [1] for demonstration):

Proposition 2.1. For each sensor $j \in[1 \mathrm{~N}]$, let $Z_{k+1}^{[j]}=$ $\left\{z_{1}^{[j]}, \ldots, z_{m[j]}^{[j]}\right\}$ be the set of current measurements produced by sensor $j$. The data update equation is given by:

$$
\begin{aligned}
& D_{k+1 \mid k+1}\left(x \mid Z^{(k+1)}\right)= \\
& \frac{\left[\frac{\delta}{\delta x}\left(\frac{\delta^{m[1]+\ldots+m[N]}}{\delta z_{1}^{[1]} \ldots \delta z_{m[N]}^{[N]}} e^{\beta\left[g^{[1]}, \ldots, g^{[N]}, h\right]}\right)\right]_{g^{[i]}=0, h=1}}{\left[\frac{\delta^{m[1]+\ldots+m[N]}}{\delta z_{1}^{[1]} \ldots \delta z_{m[N]}^{[N]}} e^{\beta\left[g^{[1]}, \ldots, g^{[N]}, h\right]}\right]_{g^{[i]}=0, h=1}}
\end{aligned}
$$

The multi-sensor data update equation (4) is generally untractable because the number of differentiated cross-terms increases dramatically with the number of sensors and/or measurements. A notable exception occurs in the single-sensor case $(N=1)$, where (4) reduces to the well-known equation developed by Mahler ([5]):

$$
\begin{aligned}
& D_{k+1 \mid k+1}\left(x \mid Z^{(k+1)}\right)= \\
& \left(1-p_{d}(x)+\sum_{z \in Z_{k+1}} \frac{p_{d}(x) L_{z}(x)}{\lambda c(z)+D_{k+1 \mid k}\left[p_{d} L_{z}\right]}\right) D_{k+1 \mid k}\left(x \mid Z^{(k)}\right)
\end{aligned}
$$

When expanded in the two-sensor case, (4) also matches Mahler's results ([7], [8]).

\subsection{Simplification by state and sensor partitioning}

Should the sensors' FOVs be such that they do not all overlap with each other, a significant waste in computational resources would occur in the implementation of (4) because many cross-terms are likely to vanish ([1]). Hence the proposed partitions of the sensors and the state space:

Definition 2.2. For any sensor $j \in[1 N]$, let $F_{k+1}^{[j]} \subset \mathcal{X}$ denote its field of view at time $k+1$ defined as:

$$
\forall x \in \mathcal{X}, x \in F_{k+1}^{[j]} \Leftrightarrow p_{d, k+1}^{[j]}(x) \neq 0
$$

Define the equivalence relation "cross" $(\leftrightarrow)$ between sensors as:

$$
\forall i, j \in[1 N],(i \leftrightarrow j) \Leftrightarrow\left(F_{k+1}^{[i]} \cap F_{k+1}^{[j]} \neq \emptyset\right)
$$

Let $\left\{P_{S}(p)\right\}_{p=1}^{P}$ be the sensor partition of $[1 N]$ formed by the equivalence classes of the transitive closure of the "cross" relation. Let $\left\{P_{T}(p)\right\}_{p=0}^{P}{ }^{5}$ be the space partition of the state space $\mathcal{X}$ defined by:

$$
P_{T}(p)=\left\{\begin{array}{cc}
\overline{\bigcup_{j=1}^{N} F_{k+1}^{[j]}} & (p=0) \\
\bigcup_{j \in P_{S}(p)} F_{k+1}^{[j]} & (p \neq 0)
\end{array}\right.
$$

Finally, for any element $P_{S}(p)$ ot the sensor partition, let $n_{p}=$ $\left|P_{S}(p)\right|$ denotes the number of sensors in $P_{S}(p)$, and let $p_{1}, \ldots, p_{n_{p}}$ denote the increasing indexes in $\left[\begin{array}{ll}1 & N\end{array}\right]$ of sensors belonging to $P_{S}(p)$.

\footnotetext{
${ }^{5}\left\{P_{S}(p)\right\}_{p=1}^{P}=\left\{P_{S, k+1}(p)\right\}_{p=1}^{P_{k+1}} \quad$ and $\quad\left\{P_{T}(p)\right\}_{p=0}^{P}=$ $\left\{P_{T, k+1}(p)\right\}_{p=0}^{P_{k+1}}$, time subscripts are omitted for simplicity's sake.
} 
Note that any sensor whose current FOV is void must be discarded when constructing $P_{S}$; likewise, $P_{T}(0)$ must be discarded if empty. Then one can show ([1]) that equation (4) can be simplified and is equivalent to:

$$
\begin{aligned}
& D_{k+1 \mid k+1}\left(x \mid Z^{(k+1)}\right)=
\end{aligned}
$$

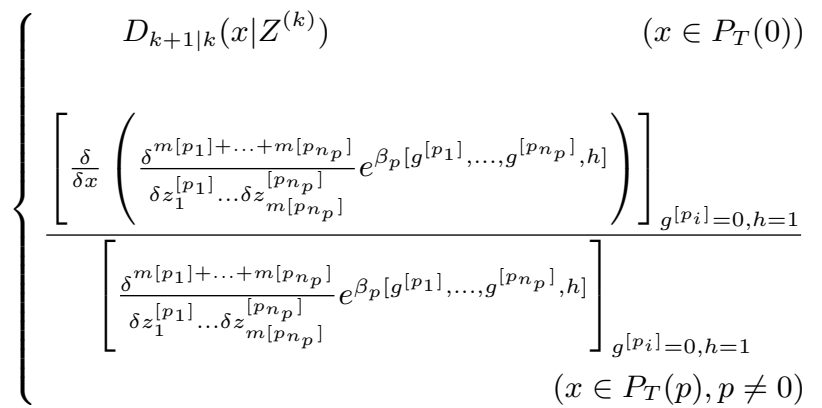

where $\beta_{p}$ is the cross-term restricted to sensors $j \in P_{S}(p) \subset[1 N]$ and to the subregion $P_{T}(p) \subset \mathcal{X}$. Since the set derivatives in (9) involve less measurements and a "smaller" cross-term $\beta_{p}$ for each partition element $p$ than the "Brute Force" data update (4), provided that the partitioning itself is not too costly, implementing the Partition method is likely to yield the true data updated density (since (4) and (9) are equivalent) with a reduced computational cost.

\section{SIMULATION}

\subsection{Scenario description}

A target state $\mathbf{x} \in \mathbb{R}^{4}$ is composed of position $(x, y)$ and velocity ( $\dot{x}$, $\dot{y}$ ) variables. Targets evolve according to a nearly constant velocity (NCV) model. The birth Process is Poisson with a constant rate, new targets are spread uniformly in the state space. Targets die whenever reaching the edges of the 2-D position subspace. The test scenario lasts 400 time steps and involves 12 targets, the targets' positions are depicted in figures 1 and 2 .

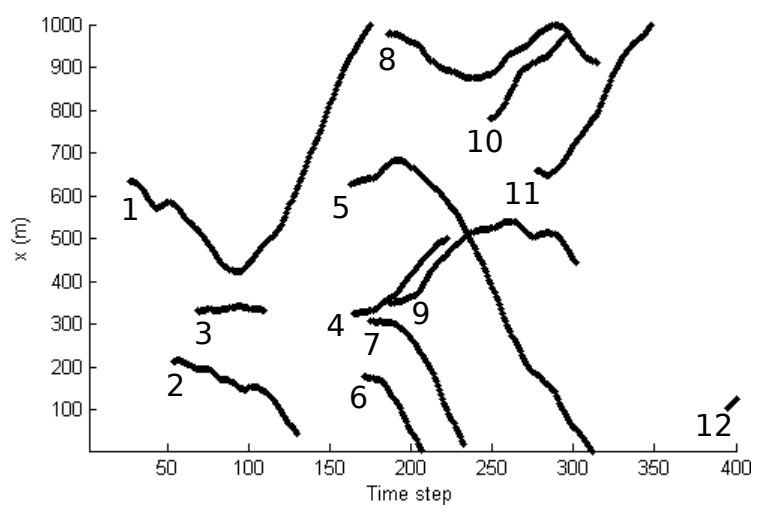

Fig. 1: Targets' positions along $\mathrm{x}$-axis

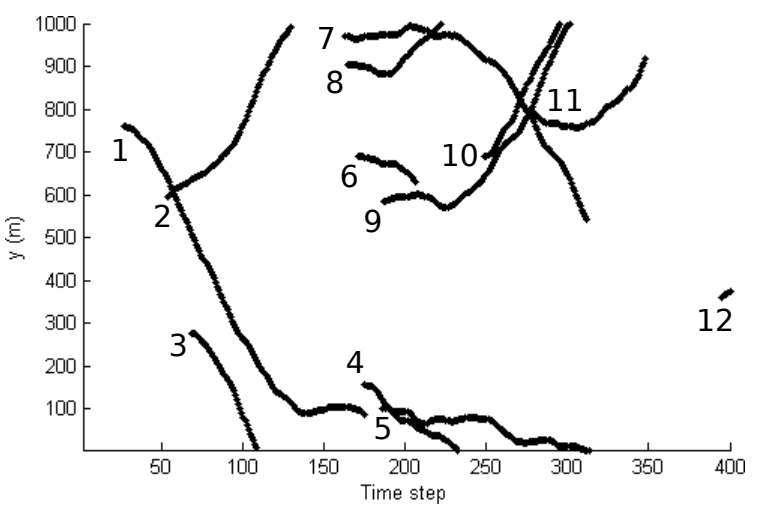

Fig. 2: Targets' positions along y-axis

The five sensors provide measurements with an independant Gaussian noise on each variable $x, y, \dot{x}$ and $\dot{y}$ and false alarms uniformly spread inside their FOV. Each sensor has its own set of sensing parameters (detection probability, FOV shape, false alarm rate, noise variances). Their FOVs are assumed fixed and spread as follows:

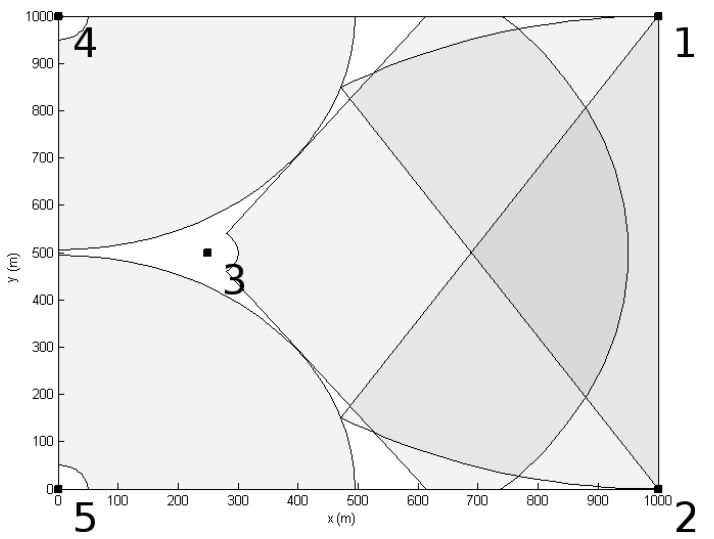

Fig. 3: Sensors' positions (dots) and FOVs in position subspace

The PHD multi-target tracker was implemented with a particle filter ([9]), in this particular case the "cross" relation (7) is restricted as follows: two sensors $j_{1}, j_{2}$ cross each other if and only if at least one particle $\tilde{\mathbf{x}^{i}}$ belongs to both FOVs. Note (fig. 3) that sensors 4 and 5 have isolated FOVs; therefore, each one is a singleton in the sensor partition regardless of the current spread of the particles in the state space. Sensors 1, 2 and 3, on the other hand, may be in the same partition element or split in several elements at each time step.

\subsection{Results}

The following results were averaged over a batch of 10 runs from the scenario described above. At every time step, the PDH $D_{k+1 \mid k}$ was data updated with the Brute Force method (4) and the Partition method (9). As expected, the data updated densities with the two methods were identical; the computed Kullback-Leibler distance between the two udpated PHDs remainded around $10^{-16}$ during the whole scenario.

Figure 4 shows that the computational cost of the data update is likely to increase around time steps 200 and 280 . Indeed, the estimated target number is high and several targets, evolving in the 
subregion covered by sensors 1,2 and 3 , are likely to generate several measurements each.

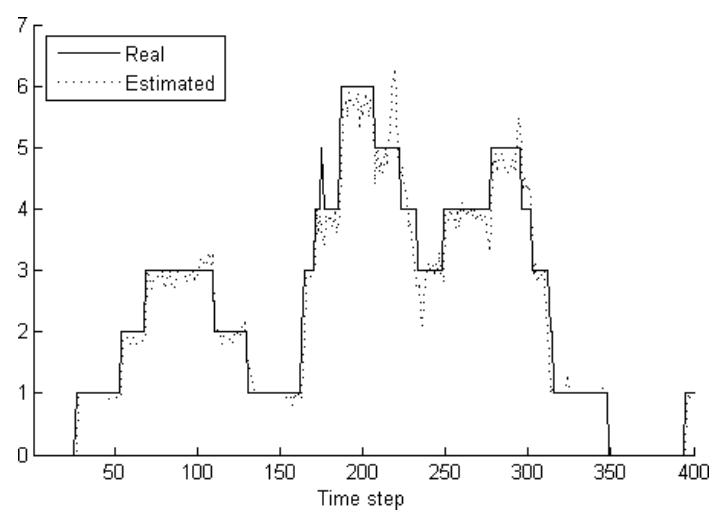

Fig. 4: Target number (real and estimated)

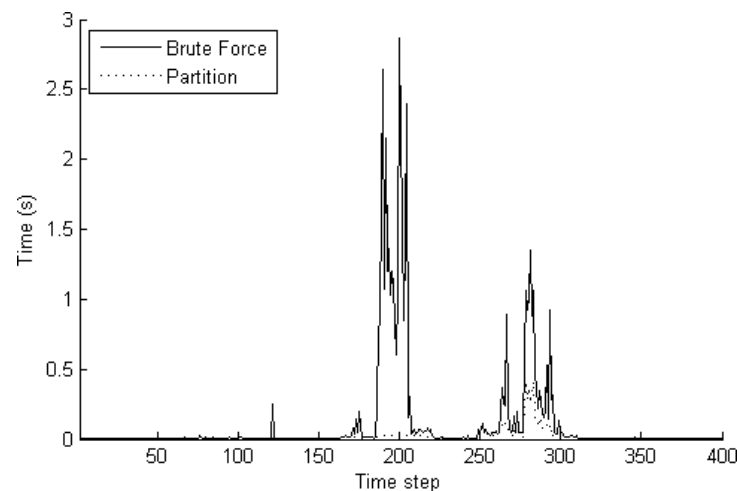

Fig. 5: Computing time of Brute Force and Partition methods

Figure 5 clearly shows that the computational cost of the Brute Force method increased dramatically around both time steps 200 and 280 , while the cost of the Partition method remained significantly lower.

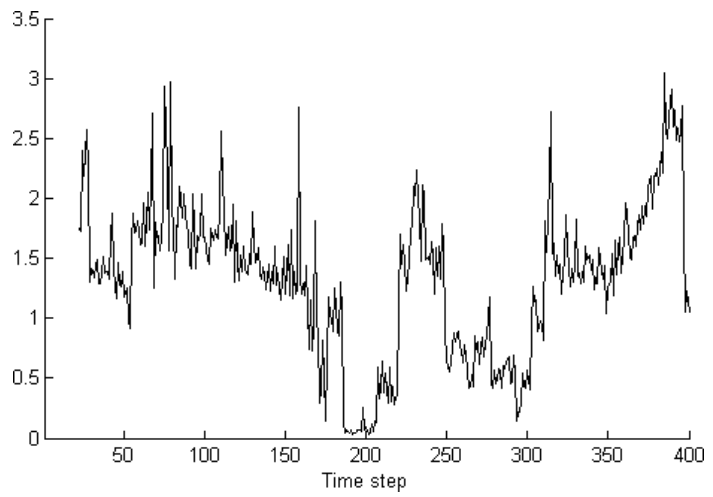

Fig. 6: Computing time ratio (Partition over Brute Force)

Figure 6 shows that the Partition method was most effective around time step 200 where, according to the target positions (fig. 1 and 2), targets were spread all over the state space. It is likely that the particules were also spread all over the state space, therefore increasing the impact of the partioning. Figure 6 also shows that the cost of the partitioning itself may be greater than the cost reduction of the data update, in which case the Partition method was less efficient than the Brute Force method; however the cost incurred by the Partition method remained low enough (fig. 5). In overall, the computational gain of the Partition method on a run of the test scenario was around 35 seconds.

\section{CONCLUSION AND FURTHER WORK}

In this paper a generalization of the single-sensor PHD data update equation to the multi-sensor case is proposed. Then, a joint sensors and state space partitioning is proposed in order to lighten the computational cost of its implementation. Essentialy, the Partition method shows that the data update can be processed independently in each partition element of the state space. This allows the design of PHD multi-sensor multi-target tracker which provides the true data updated density with a lighter computational cost, depending on the configuration of the sensors' FOVs.

The Partition method may have other practical applications that could be explored in further works. The first lead would be to apply either a true data update or a iterated-corrector approximation ([8]) independently on each partitioned element $P_{T}(p)$ of the target space, depending on the number of sensors and their related measurements in the corresponding sensor partition element $P_{S}(p)$. This method could lead to an interesting compromise between the cost-effective iterated-corrector approximation and the untractable true multi-sensor update. Another lead would be to apply a similar partitioning technique in order to obtain a tractable PENT-based ([6]) multi-sensor manager.

\section{REFERENCES}

[1] E. Delande, E. Duflos, D. Heurguier, and P. Vanheeghe. Multitarget phd filtering: proposition of extensions to the multi-sensor case. Research Report RR-7337, INRIA, July 2010.

[2] R. Helmick. Multitarget-Multisensor Tracking: Applications and Advances (Volume III), chapter 3. Artech House, 2000.

[3] C. M. Kreucher, K. D. Kastella, and A. O. Hero III. Multitarget tracking using the joint multitarget probability density. IEEE Transactions on Aerospace and Electronic Systems, 41:13961414, 2005.

[4] R. P. S. Mahler. Handbook of Multisensor Data Fusion, chapter 14. CRC Press, 2002.

[5] R. P. S. Mahler. Multitarget bayes filtering via first-order multitarget moments. IEEE Transactions on Aerospace and Electronic Systems, 39(4):1152-1178, October 2003.

[6] R. P. S. Mahler. Sensor management with non-ideal sensor dynamics. In Information Fusion, 2004, Proceedings of the 7th International Conference on, June 2004

[7] R. P. S. Mahler. The multisensor phd filter, i: General solution via multitarget calculus. In Signal Processing, Sensor Fusion, and Target Recognition XVIII, Proceedings of SPIE, April 2009.

[8] R. P. S. Mahler. The multisensor phd filter, ii: Erroneous solution via 'Poisson magic'. In Signal Processing, Sensor Fusion, and Target Recognition XVIII, Proceedings of SPIE, April 2009.

[9] B-N. Vo, S. Singh, and A. Doucet. Sequential monte carlo methods for multi-target filtering with random finite sets. IEEE Transactions on Aerospace and Electronic Systems, 41(4):12241245, October 2005. 